Ibn Al-Haitham Jour. for Pure \& Appl. Sci. 33 (2) 2020

Ibn Al Haitham Journal for Pure and Applied Science

Journal homepage: http://jih.uobaghdad.edu.iq/index.php/j/index

\title{
Interval Value Fuzzy $k$-Ideals of a KU-Semigroup
}

Sally A. Talib

Department of Mathematics, College of Education for Pure Science Ibn-Al-Haitham, University of Baghdad, Baghdad, Iraq.

sallyabdulkarim89@gmail.com

fa sa20072000@yahoo.com

Article history: Received 23 June 2019, Accepted 6 August 2019, Published in April 2020.

Doi: $10.30526 / 33.2 .2430$

\begin{abstract}
The notion of interval value fuzzy $k$-ideal of KU-semigroup was studied as a generalization of afuzzy $k$-ideal of KU-semigroup. Some results of this idea under homomorphism are discussed. Also, we presented some properties about the image (preimage) for interval valued fuzzy $k$-ideals of a KU-semigroup. Finally, the product of interval valued fuzzyk-ideals is established.
\end{abstract}

Keywords: KU-algebra;KU-semigroup; interval valuefuzzy $S$-ideal; interval value fuzzy $k$ ideal; interval value fuzzy $P$-ideal.

\section{Introduction}

Prabpayak and Leerawat [1,2]. Introduced the KU-algebra which is dual of BCK-algebra. In [3]. Kareem and Hasan introduced the KU-semigroups and defined some types of ideals in this concept. The fuzzy set was initiated by Zadeh, in [4]. Since then this concept has been applied in many distinct branches of mathematics such as groups, vector space, topological space and ring theory. In [5]. The idea of fuzzy KU-algebra was introduced by Mostafa et al. and the fuzzy KU-semigroupwas studied by Elaf and Kareem in [6]. Fuzzy sets extensions such as intuitionistic fuzzy sets, Bipolar-valued fuzzy sets, and interval valued fuzzy sets were studied by many mathematicians see [7-12]. The notion of interval value fuzzy $k$-ideal of KUsemigroup was studied in this paper and few properties were investigated. Some results of these ideals ina KU-semigroup under homomorphism are discussed. The image of these ideals in a KU-semigroup was defined. Finally, the product of some ideals was established.

\section{Preliminaries}

In this part, we review some concepts related to KU-semigroup and interval valued fuzzy logic.

Definition (1) [1-2]. Algebra $(\boldsymbol{N}, *, 0)$ is called a KU-algebra if, for all $\chi, \gamma, \tau \in \mathcal{N}$, 
$\left(\mathrm{ku}_{1}\right)(\chi * \gamma) *[(\gamma * \tau) *(\chi * \tau)]=0$,

$\left(\mathrm{ku}_{2}\right) \chi * 0=0$,

$\left(\mathrm{ku}_{3}\right) 0^{*} \chi=\chi$,

$\left(\mathrm{ku}_{4}\right) \chi^{*} \gamma=0$ and $\gamma * \chi$ implies $\chi=\gamma$ and,

$\left(\mathrm{ku}_{5}\right) \chi * \chi=0$.

A binary relation $\leq$ on $\boldsymbol{N}$ is defined by $\chi \leq \gamma \Leftrightarrow \gamma * \chi=0$. It follows that $(\mathrm{N}, \leq)$ is a partially ordered set. Then, $(\aleph, *, 0)$ satisfies the following statements. For all $\chi, \gamma, \tau \in \aleph$,

$\left(\mathrm{Ku}_{\grave{1}}\right)[(\gamma * \tau) *(\chi * \tau)] \leq(\chi * \gamma)$

$\left(\mathrm{Ku}_{\grave{2}}\right) 0 \leq \chi$,

$\left(\mathrm{Ku}_{3}\right) \chi \leq \gamma, \gamma \leq \chi$ implies $\chi=\gamma$

$\left(\mathrm{Ku}_{4}\right) \gamma * \chi \leq \chi$.

Example (2)[1]. Let $\aleph=\{0, a, b, c\}$ be a set and $*$ a binary operation defined in the following table

\begin{tabular}{|c|c|c|c|c|}
\hline$*$ & 0 & $a$ & $b$ & $c$ \\
\hline 0 & 0 & $a$ & $b$ & $c$ \\
\hline$a$ & 0 & 0 & 0 & $b$ \\
\hline$b$ & 0 & $b$ & 0 & $a$ \\
\hline$c$ & 0 & 0 & 0 & 0 \\
\hline
\end{tabular}

It is easy to see that $(\aleph, *, 0)$ is a KU-algebra.

Theorem(3) [2]. Let $(\aleph, *, 0)$ be a KU-algebra. Then, for all $\chi, \gamma, \tau \in \aleph$,

(1) If $\chi \leq \gamma$ implies $\gamma * \tau \leq \chi * \tau$,

(2) $\chi *(\gamma * \tau)=\gamma *(\chi * \tau)$,

(3) $((\gamma * \chi) * \chi) \leq \gamma$.

Definition (4) [1-2]. Let $(\aleph, *, 0)$ be a KU-algebra and $I$ be a non- empty subset of $\aleph$. Then $I$ is called an ideal of $\boldsymbol{N}$ if for any $\chi, \gamma \in \aleph$, then

(i) $\quad 0 \in$ Iand

(ii) if $\chi * \gamma \in I$ and $\chi \in I$ imply $\gamma \in I$.

Definition (5) [1-2]. Let $I$ be a subset of a KU-algebra $(\aleph, *, 0)$ and $\neq \varphi$. Then $I$ is named a KU-ideal of $\aleph$, if

$\left(\mathrm{I}_{1}\right) 0 \in I$ and

$\left(\mathrm{I}_{2}\right) \forall \chi, \gamma, \tau \in \aleph,(\chi *(\gamma * \tau)) \in I$ and $\gamma \in$ Iimply $\chi * \tau \in I$.

Definition (6)[3]. A KU-semigroup is a nonempty set $\aleph$ with two binary operations $*$,oand a constant 0 satisfying the following

(I) $(\aleph, *, 0)$ is a KU-algebra, 
(II) $(\mathrm{N}, \mathrm{o})$ is a semigroup,

(III) The operation $\circ$ is distributive (on both sides) over theoperation *, i.e.

$\chi \circ(\gamma * \tau)=(\chi \circ \gamma) *(\chi \circ \tau)$ and $(\chi * \gamma) \circ \tau=(\chi \circ \tau) *(\gamma \circ \tau)$, forall $\chi, \gamma, \tau \in X$.

Example (7)[3]. Let $\aleph=\{0,1,2,3\}$ be a set. Define* -operation and o-operationby the following tables

\begin{tabular}{|c|c|c|c|c|}
\hline$*$ & 0 & 1 & 2 & 3 \\
\hline 0 & 0 & 1 & 2 & 3 \\
\hline 1 & 0 & 0 & 0 & 2 \\
\hline 2 & 0 & 2 & 0 & 1 \\
\hline 3 & 0 & 0 & 0 & 0 \\
\hline
\end{tabular}

\begin{tabular}{|c|c|c|c|c|}
\hline$\circ$ & 0 & 1 & 2 & 3 \\
\hline 0 & 0 & 0 & 0 & 0 \\
\hline 1 & 0 & 1 & 0 & 1 \\
\hline 2 & 0 & 0 & 2 & 2 \\
\hline 3 & 0 & 1 & 2 & 3 \\
\hline
\end{tabular}

Then $(\aleph, *, \circ, 0)$ is a KU-semigroup.

Definition (8)[3]. A subKU-semigroup is a non-empty subset $A$ of a KU-semigroup $N$ and it is satisfied $\chi * \gamma, \chi \circ \gamma \in \mathrm{A}$, for all $\chi, \gamma \in \mathrm{A}$.

Definition (9)[3]. Let $(\aleph, *, \circ, 0)$ be a KU-semigroup and $\varphi \neq I \subseteq \aleph$. Then,

$I$ is named an $S$-ideal of $\aleph$, if

i) $\quad I$ is an ideal of a KU-algebra $(\aleph, *, 0)$,

ii) For all $\chi \in \aleph, a \in I$, we have $\chi \circ a \in I$ and $a \circ \chi \in I$.

Definition (10)[3]. Let $(\aleph, *, \circ, 0)$ be a KU- semigroup and $\varphi \neq A \subseteq \aleph$. Then $\mathrm{A}$ is said to be a $k$-ideal of $\aleph$, if

i) $\mathrm{A}$ is an $\mathrm{KU}$-ideal of a $\mathrm{KU}$ - algebra $(\aleph, *, 0)$,

ii) For all $\chi \in X, a \in \mathrm{A}$, we have $\chi \circ a \in \mathrm{A}$ and $a \circ \chi \in \mathrm{A}$.

Definition (11)[3]. Let $(\aleph, *, \circ, 0)$, be a KU- semigroup and $\varphi \neq A \subseteq \aleph$. Then,

A is said to be a $P$-idealof $\aleph$, if

(p1) For any $\chi, \gamma, \tau \in \mathcal{N}, \tau *(\chi * \gamma) \in \mathrm{A}$ and $\tau * \chi \in \mathrm{A} \Rightarrow \tau * \gamma \in \mathrm{A}$.

( $\left.\mathrm{p}_{2}\right)$ For all $\chi \in \aleph, a \in \mathrm{A}$, we have $\chi \circ a \in \mathrm{A}$ and $a \circ \chi \in \mathrm{A}$.

Definition (12)[3]. Let $\aleph$ and $\aleph^{\prime}$ be two KU-semigroups. A mapping $f: \aleph \rightarrow \aleph^{\prime}$ is called a KU-semigroup homomorphism if $f(\chi * \gamma)=f(\chi) * f(\gamma)$ and $f(\chi \circ \gamma)=f(\chi) \circ f(\gamma)$, for all $\chi, \gamma \in \aleph$.

The kernel of $\mathrm{f}$ is denoted by ker $\mathrm{f}$ and is defined by $\{\chi \in \mathrm{\aleph}: \mathrm{f}(\chi)=0\}$. Moreover, the image of $\mathrm{f}$ is denoted by $i m f$ and is defined by $\left\{f(\chi) \in \aleph^{\prime}: \chi \in \aleph\right\}$.

We review some concepts of fuzzy logic.

A function $\mu: \aleph \rightarrow[0,1]$ is said to be a fuzzy set of a set $\aleph$ and the set $U(\mu, t)=\{\chi \in \aleph: \mu(\chi) \geq t\}$ is said to be a level set of $\mu$, for $t$ in $[0,1]$. 
Definition (13)[6]. A fuzzy set $\mu$ of $\aleph$ is called a fuzzy sub KU-semigroup if, for all $\chi, \gamma \in \aleph$

i) $\quad \mu(\chi * \gamma) \geq \min \{\mu(\chi), \mu(\gamma)\}$,

ii) $\quad(\chi \circ \gamma) \geq \min \{\mu(\chi), \mu(\gamma)\}$.

Definition (14)[6]. A fuzzy set $\mu$ in $\aleph$ is called a fuzzy $S$-ideal of $\aleph$ if, for all $\chi, \gamma \in \aleph$.

$\left(S_{1}\right) \mu(0) \geq \mu(\chi)$,

$\left(S_{2}\right) \mu(\gamma) \geq \min \{\mu(\chi * \gamma), \mu(\chi)\}$

$\left.\left(S_{3}\right) \mu(\chi \circ \gamma)\right) \geq \min \{\mu(\chi), \mu(\gamma)\}$.

Definition (15)[6]. A fuzzy set $\mu$ in $\aleph$ is called a fuzzy $k$-ideal of $N$ if it satisfies the following conditions: for all $\chi, \gamma, \tau \in \aleph$.

$\left(k_{1}\right) \mu(0) \geq \mu(\chi)$,

$\left(k_{2}\right) \mu(\chi * \tau) \geq \min \{\mu(\chi *(\gamma * \tau), \mu(\gamma)\}$

$\left.\left(k_{3}\right) \mu(\chi \circ \gamma)\right) \geq \min \{\mu(\chi), \mu(\gamma)\}$.

Example (16)[6]. Let $\aleph=\{0, a, b, c, d\}$ be a set. Define* - operation and o- operation by the following tables

\begin{tabular}{|c|c|c|c|c|c|}
\hline$*$ & 0 & $\mathrm{a}$ & $\mathrm{b}$ & $\mathrm{c}$ & $\mathrm{d}$ \\
\hline 0 & 0 & $\mathrm{a}$ & $\mathrm{b}$ & $\mathrm{c}$ & $\mathrm{d}$ \\
\hline $\mathrm{a}$ & 0 & 0 & $\mathrm{~b}$ & $\mathrm{c}$ & $\mathrm{d}$ \\
\hline $\mathrm{b}$ & 0 & $\mathrm{a}$ & 0 & $\mathrm{c}$ & $\mathrm{d}$ \\
\hline $\mathrm{c}$ & 0 & $\mathrm{a}$ & 0 & 0 & $\mathrm{~d}$ \\
\hline $\mathrm{d}$ & 0 & 0 & 0 & 0 & 0 \\
\hline
\end{tabular}

\begin{tabular}{|c|c|c|c|c|c|}
\hline$\circ$ & 0 & $\mathrm{a}$ & $\mathrm{b}$ & $\mathrm{c}$ & $\mathrm{d}$ \\
\hline 0 & 0 & 0 & 0 & 0 & 0 \\
\hline $\mathrm{a}$ & 0 & 0 & 0 & 0 & 0 \\
\hline $\mathrm{b}$ & 0 & 0 & 0 & 0 & $\mathrm{~b}$ \\
\hline $\mathrm{c}$ & 0 & 0 & 0 & $\mathrm{~b}$ & $\mathrm{c}$ \\
\hline $\mathrm{d}$ & 0 & $\mathrm{a}$ & $\mathrm{b}$ & $\mathrm{c}$ & $\mathrm{d}$ \\
\hline
\end{tabular}

Then $(\aleph, *, \circ, 0)$ is a KU-semigroup. Define a fuzzy set $\mu: \aleph \rightarrow[0,1]$ by $\mu(0)=\mu(a)=0.4, \mu(b)=\mu(c)=0.2, \mu(d)=0.1$. Then by routine calculation we can prove that $\mu$ is afuzzy $k$-ideal ofN.

Definition (17) [6]. The Cartesian product of two fuzzy sets $\mu$ and $\beta$ of $\aleph$ is denoted by $\mu \times \beta: \aleph \times \aleph \rightarrow[0,1]$ and defined by $(\mu \times \beta)(\chi, \gamma)=\min \{\mu(\chi), \beta(\gamma)\}, \forall \chi, \gamma \in \aleph$.

Definition (18)[6]. Let $\mu$ be a fuzzy set in $\aleph$. If $\mu$ is defined by: $\aleph \times \aleph \rightarrow[0,1]$, then $\mu$ is said to be a fuzzy relation on a set $S$, where $\mathrm{S} \subseteq \aleph$.

Definition (19)[6]. Let $\mu$ be a fuzzy relation on $\aleph$ and $\beta$ be a fuzzy subset of $\aleph$. Then the strongest fuzzy relation on $\mathrm{N}$ is denoted by $\mu_{\beta}$ and is defined as follows

$\mu_{\beta}(\chi, \gamma)=\min \{\beta(\chi), \beta(\gamma)\}, \forall \chi, \gamma \in \kappa$.

\section{Interval value fuzzy $\boldsymbol{k}$-ideals in KU-semigroup}

In this part, we recall the definition of interval valued fuzzy set $\tilde{\mu}$ of $\aleph$ as follows 
$\tilde{\mu}=\left\{\left(\chi,\left[\mu^{L}(\chi), \mu^{U}(\chi)\right]\right): \chi \in \aleph\right\}$, by briefly $\tilde{\mu}=\left[\mu^{L}, \mu^{U}\right]$, where $\mu^{L}$ and $\mu^{U}$ are two fuzzy sets in $\aleph$ such that $\mu^{L}(\chi) \leq \mu^{U}(\chi)$, for all $\chi \in \aleph$ and the closed sub-intervals of [0,1] is denoted by $\mathrm{D}[0,1]$. Note that, if $\mu^{L}(\chi)=\mu^{U}(\chi)=c$, where $0 \leq c \leq 1$, then $\tilde{\mu}(\chi)=[c, c]$, it follows that $\tilde{\mu}(\chi) \in \mathrm{D}[0,1]$ and it is given by $\tilde{\mu}: \aleph \rightarrow D[0,1]$, for all $\chi \in \mathcal{N}$ and $\mathrm{D}[0,1]=\left\{\left[a^{L}, a^{U}\right]: a^{L} \leq a^{U}\right.$ for $\left.a^{L}, a^{U} \in[0,1]\right\}$.

Consider, two elements $D_{1}=\left[a^{L}, a^{U}\right]$ and $D_{2}=\left[b^{L}, b^{U}\right]$ in $\mathrm{D}[0,1]$ are defined by $r \min \left(D_{1}, D_{2}\right)=\left[\min \left(a^{L}, b^{L}\right), \min \left(a^{U}, b^{U}\right)\right]$ and $r \max \left(D_{1}, D_{2}\right)=\left[\max \left(a^{L}, b^{L}\right), \max \left(a^{U}, b^{U}\right)\right]$.

Definition (20). $\operatorname{Let}(\aleph, *, \circ, 0)$ be a KU- semigroup. If $\tilde{\mu}: \aleph \rightarrow D[0,1]$, then the level subset of $\tilde{\mu}$ is denoted by $\tilde{\mu}_{\tilde{t}}$ and it is defined by $\tilde{\mu}_{\tilde{t}}=\{\chi \in \aleph: \tilde{\mu}(\chi) \geq \tilde{t}\}$, for every $[0,0] \leq \tilde{t} \leq[1,1]$.

Definition (21). Let $(\aleph, *, \circ, 0)$ be a KU-semigroup and $\tilde{\mu}: \aleph \rightarrow D[0,1]$. Then $\tilde{\mu}$ is called an interval valued fuzzy sub KU- semigroup $\aleph$, if it satisfies the following conditions $\tilde{\mu}(\chi * \gamma)) \geq \operatorname{rmin}\{\tilde{\mu}(\chi), \tilde{\mu}(\gamma)\}, \tilde{\mu}(\chi \circ \gamma)) \geq \operatorname{rmin}\{\tilde{\mu}(\chi), \tilde{\mu}(\gamma)\}, \forall \chi, \gamma \in \aleph$.

Example (22). Let $\aleph=\{0,1,2,3\}$ be a set. We define two operations by the following tables

\begin{tabular}{|c|c|c|c|c|}
\hline$*$ & 0 & 1 & 2 & 3 \\
\hline 0 & 0 & 1 & 2 & 3 \\
\hline 1 & 0 & 0 & 0 & 2 \\
\hline 2 & 0 & 2 & 0 & 1 \\
\hline 3 & 0 & 0 & 0 & 0 \\
\hline
\end{tabular}

\begin{tabular}{|c|c|c|c|c|}
\hline$\circ$ & 0 & 1 & 2 & 3 \\
\hline 0 & 0 & 0 & 0 & 0 \\
\hline 1 & 0 & 1 & 0 & 1 \\
\hline 2 & 0 & 0 & 2 & 2 \\
\hline 3 & 0 & 1 & 2 & 3 \\
\hline
\end{tabular}

$\operatorname{Then}(\aleph, *, \circ, 0)$ is a KU-semigroup. Define $\tilde{\mu}(\chi)$ as follows $\tilde{\mu}(\chi)=$

$$
\begin{gathered}
\text { if } \chi=\{0,1,2\} \\
\text { if } \chi=3
\end{gathered}
$$

And by applying definition 21 , we can prove that $\tilde{\mu}(\chi)$ is an interval valued fuzzysub KUsemi group of $\aleph$.

Definition (23). $\operatorname{Let}(\aleph, *, \circ, 0)$ be a KU-semigroup and $\tilde{\mu}: \aleph \rightarrow D[0,1]$. Then $\tilde{\mu}$ is named an interval valuedfuzzy $S$-ideal of $\aleph$ if

$\left(i_{1}\right) \tilde{\mu}(0) \geq \tilde{\mu}(\chi), \forall \chi \in \aleph$,

$\left(i_{2}\right)$ For all, $\chi, \gamma \in \aleph, \tilde{\mu}(\gamma) \geq r \min \{\tilde{\mu}(\chi * \gamma), \tilde{\mu}(\chi)\}$,

$\left.\left(i_{3}\right) \tilde{\mu}(\chi \circ \gamma)\right) \geq r \min \{\tilde{\mu}(\chi), \tilde{\mu}(\gamma)\}$.

Definition (24). $\operatorname{Let}(\aleph, *, \circ, 0)$ be a KU-semigroup and $\tilde{\mu}: \aleph \rightarrow D[0,1]$. Then $\tilde{\mu}$ is named an interval valued fuzzy $k$ - ideal of $N$ if

$\left(\mathrm{f}_{1}\right) \tilde{\mu}(0) \geq \tilde{\mu}(\chi), \forall \chi \in \aleph$,

$\left(\mathrm{f}_{2}\right)$ For all $\chi, \gamma, \tau \in \aleph, \tilde{\mu}(\chi * \tau) \geq \operatorname{rmin}\{\tilde{\mu}(\chi *(\gamma * \tau), \tilde{\mu}(\gamma)\}$,

$\left.\left(f_{3}\right) \tilde{\mu}(\chi \circ \gamma)\right) \geq r \min \{\tilde{\mu}(\chi), \tilde{\mu}(\gamma)\}$. 
Example (25). From Example16, we define $\tilde{\mu}(\chi)$ as follows:

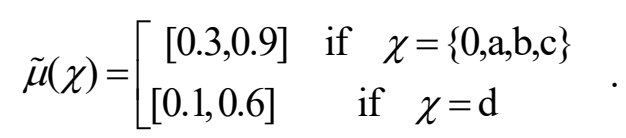

By this definition of $\tilde{\mu}$, we can prove it is an interval valued fuzzy $k$-ideal.

Theorem (26). $\operatorname{Let}(\boldsymbol{N}, *, \circ, 0)$ be a KU-semigroup. Then $\tilde{\mu}$ in $\aleph$ is an interval valued fuzzy $k$ ideal if and only if it is an interval valued fuzzy $S$ - ideal of $\aleph$.

Proof. $(\Rightarrow)$ By taking $\chi=0$ in $\left(\mathrm{f}_{2}\right),\left(\mathrm{f}_{3}\right)$ and using $\left(\mathrm{ku}_{3}\right)$, we obtain $\forall \gamma, \tau \in \mathrm{N}$ $\tilde{\mu}(\tau)=\tilde{\mu}(0 * \tau) \geq \operatorname{rmin}\{\tilde{\mu}(0 *(\gamma * \tau)), \tilde{\mu}(\gamma)\}=\operatorname{rmin}\{\tilde{\mu}(\gamma * \tau), \tilde{\mu}(\gamma)\}$ and $\tilde{\mu}(\chi \circ \gamma)) \geq r \min \{\tilde{\mu}(\chi), \tilde{\mu}(\gamma)\}$ are satisfied.

$(\Leftarrow)$ we have $\tilde{\mu}(\chi * \tau) \geq r \min \{\tilde{\mu}(\gamma *(\chi * \tau)), \tilde{\mu}(\gamma)\}$ and by apply Theorem 3 , we get $\tilde{\mu}(\chi * \tau) \geq \operatorname{rmin}\{\tilde{\mu}(\chi *(\gamma * \tau)), \tilde{\mu}(\gamma)\}$ and by Definition 24, we have $\tilde{\mu}(\chi \circ \gamma)) \geq r \min \{\tilde{\mu}(\chi), \tilde{\mu}(\gamma)\}$. Thus $\tilde{\mu}$ is an interval valuedfuzzy $k$-ideal of $\aleph$.

Theorem (27). Let $(\aleph, *, \circ, 0)$ be a KU-semigroup, $A$ be a nonempty subset of $\aleph$ and $\tilde{\mu}$ be an interval valued fuzzy set in $\aleph$. We define $\tilde{\mu}$ as follows $\tilde{\mu}(\chi)=\left\{\begin{array}{l}{\left[t_{1}, t_{2}\right]} \\ {\left[\alpha_{1}, \alpha_{1}\right]}\end{array} \quad \begin{array}{c}\text { otherwise }\end{array} \quad\right.$ where $t_{1}>\alpha_{1}, t_{2}>\alpha_{2}$ and $\alpha_{1}, \alpha_{1}, t_{1}, t_{2} \in \mathrm{D}[0,1] . \quad$ Then $A$ is $a k$-ideal of $\aleph$ if and only if $\tilde{\mu}$ is an interval valued fuzzy $k$-ideal of $\aleph$. Moreover, $\aleph_{\tilde{\mu}}=\mathrm{A}$, such that $\aleph_{\tilde{\mu}}=\{\chi \in \mathrm{N}: \tilde{\mu}(\chi)=\tilde{\mu}(0)\}$.

Proof. $(\Leftarrow)$ Since $\tilde{\mu}(0) \geq \tilde{\mu}(\chi), \forall \chi \in \aleph$, we get $\tilde{\mu}(0)=\left[t_{1}, t_{2}\right]$, then $0 \in \mathrm{A}$.

$\operatorname{Let}(\chi *(\gamma * \tau)) \in \mathrm{A}$ and $\gamma \in \mathrm{A}$, for any $\chi, \gamma, \tau \in \mathrm{K}$. Using $\left(f_{2}\right)$, we have $\tilde{\mu}(\chi * \tau) \geq \operatorname{rmin}\{\tilde{\mu}(\chi *(\gamma * \tau)), \tilde{\mu}(\gamma)\}=\operatorname{rmin}\left\{\left[t_{1}, t_{2}\right],\left[t_{1}, t_{2}\right]\right\}=\left[t_{1}, t_{2}\right]$ and thus $\tilde{\mu}(\chi * \tau)\}=\left[t_{1}, t_{2}\right]$, it follows that $\chi * \tau \in \mathrm{A}$.

Now, let $\chi \in A$ and $\gamma \in A$ by using $\left(f_{3}\right)$, we know that $\tilde{\mu}(\chi \circ \gamma) \geq r \min \{\tilde{\mu}(\chi), \tilde{\mu}(\gamma)\}=r \min \left\{\left[t_{1}, t_{2}\right],\left[t_{1}, t_{2}\right]\right\}=\left[t_{1}, t_{2}\right]$, and thus $\tilde{\mu}(\chi \circ \gamma)=\left[t_{1}, t_{2}\right]$. Hence $\chi \circ \gamma \in A$ and it follows that $A$ is a $k$-ideal of $\aleph$. $(\Rightarrow)$ Since $0 \in A$, it follows that $\tilde{\mu}(0)=\left[t_{1}, t_{2}\right] \geq \tilde{\mu}(\chi)$ for all $\chi \in \aleph$. Let $\chi, \gamma, \tau \in \aleph$. If $\gamma \notin \mathrm{A}$ and $\chi * \tau \in \mathrm{A}$, then clearly $\tilde{\mu}(\chi * \tau) \geq \operatorname{rmin}\{\tilde{\mu}(\chi *(\gamma * \tau), \tilde{\mu}(\gamma)\}$.

Assume that $\gamma \in A$ and $\chi * \tau \notin A$. Then by definition9, we have $\chi *(\gamma * \tau) \notin A$. Therefore $\tilde{\mu}(\chi * \tau)=\left[\alpha_{1}, \alpha_{1}\right]=\operatorname{rmin}\{\tilde{\mu}(\chi *(\gamma * \tau), \tilde{\mu}(\gamma)\}$.

Also, let $\chi \in A, \gamma \notin A$ and $\chi \circ \gamma \in A$, then clearly: $\tilde{\mu}(\chi \circ \tau)=\left[t_{1}, t_{2}\right] \geq r \min \{\tilde{\mu}(\chi), \tilde{\mu}(\gamma)\}$.

Assume that $\chi \notin A, \gamma \in \mathrm{A}$ and $\chi \circ \gamma \notin A$. Then $\tilde{\mu}(\chi \circ \gamma)=\left[\alpha_{1}, \alpha_{2}\right]=r \min \{\tilde{\mu}(\chi), \tilde{\mu}(\gamma)\}$.

Hence $\tilde{\mu}$ is a fuzzy $k$-ideal in $\aleph$. 
Finally, we have $\aleph_{\tilde{\mu}}=\{\chi \in \aleph: \tilde{\mu}(\chi)=\tilde{\mu}(0)\}=\left\{\chi \in \aleph: \tilde{\mu}(\chi)=\left[t_{1}, t_{2}\right]\right\}=\mathrm{A}$.

Theorem (28). $\operatorname{Let}(\aleph, *, \circ, 0)$ be a KU-semigroup and $\tilde{\mu}: \aleph \rightarrow D[0,1]$. Then the level set $\tilde{\mu}_{\tilde{t}}$ of $\tilde{\mu}$ is a $k$-ideal in $\aleph$ iff $\tilde{\mu}$ is an interval valued fuzzy $k$-ideal.

Proof. $(\Leftarrow)$ For any $\tilde{t}=\left[t_{1}, t_{2}\right] \in \mathrm{D}[0,1]$, assume $\tilde{\mu}_{\tilde{t}}$ is a non empty, then there exists $\chi \in \tilde{\mu}_{\tilde{t}}$ and $\tilde{\mu}(\chi) \geq \tilde{t}$. It follows from Definition 24 that $\tilde{\mu}(0) \geq \tilde{\mu}(\chi) \geq \tilde{t}$, so that $0 \in \tilde{\mu}_{\tilde{t}}$.

Let $\chi, \gamma, \tau \in \aleph$ such that $(\chi *(\gamma * \tau)) \in \tilde{\mu}_{\tilde{t}}$ and $\gamma \in \tilde{\mu}_{\tilde{t}}$. We have $\tilde{\mu}(\chi *(\gamma * \tau)) \geq \tilde{t}$ and $\tilde{\mu}(\gamma) \geq$ $\tilde{t}$. From Definition 24, we get the following $\tilde{\mu}(\chi * \tau) \geq \operatorname{rmin}\{\tilde{\mu}(\chi *(\gamma * \tau)), \tilde{\mu}(\gamma)\} \geq r \min [\tilde{t}, \tilde{t}]=\tilde{t}\}$, thus $\chi * \tau \in \tilde{\mu}_{\tilde{t}}$.

Now, let $a \in \tilde{\mu}_{\tilde{t}}$ and $\chi \in \aleph$, then $\tilde{\mu}(\chi) \geq \tilde{t}$ and $\tilde{\mu}(a) \geq \tilde{t}$. We get $\tilde{\mu}(\chi \circ a) \geq$ $\operatorname{rmin}\{\tilde{\mu}(\chi), \tilde{\mu}(a)\} \geq[\tilde{t}, \tilde{t}]=\tilde{t}$, which implies that $\chi \circ a \in \tilde{\mu}_{\tilde{t}}$.

Similarly, $a \circ \chi \in \tilde{\mu}_{\tilde{t}}$. Therefore $\tilde{\mu}_{\tilde{t}}$ is a $k$-ideal of $\aleph$

$(\Longrightarrow)$ Let $\tilde{\mu}_{\tilde{t}}$ be a non-empty and a $k$-ideal of $\aleph$, we have $\tilde{\mu}(\chi)=\tilde{t}$, for every $\tilde{t} \in \mathrm{D}[0,1]$ and for any $\chi \in \aleph$. This implies that $\chi \in \tilde{\mu}_{\tilde{t}}$. And since $0 \in \tilde{\mu}_{\tilde{t}}$, then $\tilde{\mu}(0) \geq \tilde{t}=\tilde{\mu}(\chi)$.

Now, we show that $\tilde{\mu}$ satisifies $\left(k_{3}\right)$ and $\left(k_{2}\right)$. If not, suppose that $\exists l, m, n \in \aleph$ such that $\tilde{\mu}(l * n) \geq r \min \{\tilde{\mu}(l *(m * n)), \tilde{\mu}(m)\}$.

put $\tilde{t}_{0}=\frac{1}{n}(\tilde{\mu}(l * n)+r \min \{\tilde{\mu}(l *(m * n)), \tilde{\mu}(m)\}$, for $\mathrm{n}$ any integer number,

so $\tilde{\mu}(l * n)<\tilde{t}_{0}<r \min \{\tilde{\mu}(l *(m * n)), \tilde{\mu}(m)\}$.

Implies that $(l *(m * n)) \in \tilde{\mu}_{\tilde{t}_{0}}$ and $m \in \tilde{\mu}_{\tilde{t}_{0}}$, but $l * n \notin \tilde{\mu}_{\tilde{t}_{0}}$, which implies $\tilde{\mu}_{\tilde{t}_{0}}$ is not a $k$ ideal of $\aleph$. Then, it is a contradiction.

Let $l, m \in \tilde{\mu}_{\tilde{t}}$ such that $\tilde{\mu}(l \circ m)<r \min \{\tilde{\mu}(l), \tilde{\mu}(m)\}$.

Then by taking $\tilde{t}_{0}=\frac{1}{n}\{\tilde{\mu}(l \circ m)+r \min \{\tilde{\mu}(l), \tilde{\mu}(m)\}$.

We have $\tilde{\mu}(l \circ m)<\tilde{t}_{0}<r \min \{\tilde{\mu}(l), \tilde{\mu}(m)\}$. Then, $l, m \in \tilde{\mu}_{\tilde{t}}$ but $l \circ m \notin \tilde{\mu}_{\tilde{t}}$.

It means that $\tilde{\mu}_{\tilde{t}_{0}}$ is not $k$-ideal of $\aleph$ and this is a contradiction. The proof is completed.

Definition (29). A fuzzy set $\tilde{\mu}$ in $\aleph$ is called an interval valued fuzzy $P$-ideal of $\aleph$ if, for all $\chi, \gamma, \tau \in \mathrm{N}$

$\left(P_{1}\right) \tilde{\mu}(0) \geq \tilde{\mu}(\chi)$

$\left(P_{2}\right) \tilde{\mu}(\tau * \gamma) \geq r \min \{\tilde{\mu}(\tau *(\chi * \gamma), \tilde{\mu}(\tau * \chi)\}$.

$\left.\left(P_{3}\right) \tilde{\mu}(\chi \circ \gamma)\right) \geq r \min \{\tilde{\mu}(\chi), \tilde{\mu}(\gamma)\}$.

Example30. Let $\boldsymbol{N}=\{0, a, b\}$ be a set. Define $*$-operation ando-operation by the following tables 
Ibn Al-Haitham Jour. for Pure \& Appl. Sci. 33 (2) 2020

\begin{tabular}{|c|c|c|c|}
\hline$*$ & 0 & $\mathrm{a}$ & $\mathrm{b}$ \\
\hline 0 & 0 & $\mathrm{~b}$ & $\mathrm{~b}$ \\
\hline $\mathrm{a}$ & 0 & 0 & $\mathrm{a}$ \\
\hline $\mathrm{b}$ & 0 & $\mathrm{a}$ & 0 \\
\hline
\end{tabular}

\begin{tabular}{|c|c|c|c|}
\hline$\circ$ & 0 & $\mathrm{a}$ & $\mathrm{b}$ \\
\hline 0 & 0 & 0 & 0 \\
\hline $\mathrm{a}$ & 0 & $\mathrm{a}$ & 0 \\
\hline $\mathrm{b}$ & 0 & 0 & $\mathrm{~b}$ \\
\hline
\end{tabular}

Then $(\aleph, *, \circ, 0)$ is a KU-semigroup. Define $\tilde{\mu}(\chi)$ as follows $\tilde{\mu}(\chi)=\left\{\begin{array}{l}{[0.3,0.8] \quad \chi=0} \\ {[0.2,0.4] \quad \chi \neq 0}\end{array}\right.$.

Then $\tilde{\mu}(\chi)$ is an interval valued fuzzy $P$-ideal of $\aleph$.

Theorem31. $\operatorname{Let}(\aleph, *, \circ, 0)$ be a KU-semigroup and $\tilde{\mu}: \aleph \rightarrow D[0,1]$. If $\tilde{\mu}$ is an interval valued fuzzy $P$-ideal, then it is an interval valued fuzzy $\mathrm{S}$-ideal.

Proof. By $\left(P_{2}\right)$ we get:

$\tilde{\mu}(\tau * \gamma) \geq r \min \{\tilde{\mu}(\tau *(\chi * \gamma), \tilde{\mu}(\tau * \chi)\}$, put $\tau=0$, we get:

$\tilde{\mu}(0 * \gamma) \geq r \min \{\tilde{\mu}(0 *(\chi * \gamma), \tilde{\mu}(0 * \chi)\}$

Thus $\tilde{\mu}(\gamma) \geq r \min \{\tilde{\mu}((\chi * \gamma), \tilde{\mu}(\chi)\}$.

The reverse of Theorem 31 is incorrect. The example32 shows the reverse.

Example 32. Let $\mathrm{N}=\{0, a, b\}$ be a set. Define (*-operation) and (o-operation) by the following tables

\begin{tabular}{|l|l|l|l|}
\hline$*$ & 0 & $a$ & $b$ \\
\hline 0 & 0 & $a$ & $b$ \\
\hline$a$ & 0 & 0 & $a$ \\
\hline$b$ & 0 & $b$ & 0 \\
\hline
\end{tabular}

\begin{tabular}{|c|c|c|c|}
\hline$\circ$ & 0 & $a$ & $b$ \\
\hline 0 & 0 & 0 & 0 \\
\hline$a$ & 0 & $a$ & 0 \\
\hline$b$ & 0 & 0 & $b$ \\
\hline
\end{tabular}

$\operatorname{Then}(\aleph, *, \circ, 0)$ is a KU-semigroup. Define $\tilde{\mu}(\chi)$ as follows:

$\tilde{\mu}(\chi)=\left\{\begin{array}{l}{[0.4,0.8] \quad \text { if } \chi=\{0, b\}} \\ {[0.1,0.3] \quad \text { if } \quad \chi=a}\end{array}\right.$.

We can easily prove that $\tilde{\mu}$ is an interval valued fuzzy $S$-idealof $\aleph$, but it is not an interval valued fuzzy $P$-ideal, since $\tilde{\mu}(0 * a)=[0.1,0.3] \leq r \min \{\tilde{\mu}(0 *(b * a)), \tilde{\mu}(0 * b)\}=$ $[0.4,0.8]$.

\section{Study of Image (Pre-image) for interval valued fuzzy $k$-ideal}

The image and the pre- image are important topics in modern algebra so we will focus on these two concepts in this part of our paper. We will study these concepts with the interval 
valued fuzzy $k$-ideals in a KU-semigroup $\aleph$ under homomorphism. Also, wewill prove that the products of interval valued fuzzy $k$-ideals are a fuzzy $k$-ideal of a KU-semigroup $N$.

Definition33. Let $f: \aleph \rightarrow Y$ be a mapping from KU-semigroup $\aleph$ into KU-semigroup $Y$ and $\widetilde{\mu}$ be an interval valued fuzzy subset of $\aleph$. We define the image for $\tilde{\mu}$ under $f$, denoted by $f(\tilde{\mu})$ as follows $f(\tilde{\mu})(\gamma)=\left\{\begin{array}{c}\sup \tilde{\mu}(\chi)_{\chi \in f^{-1}(\gamma)}, \\ 0 \quad \text { if } \quad f^{-1}(\gamma)=\{\chi \in \kappa: f(\chi)=\gamma\} \neq \emptyset \\ \text { otherwise }\end{array}\right.$

And the pre-image for $\tilde{\beta}$ under $f$, where $\tilde{\beta}$ is an interval valued fuzzy subset of $Y$, denoted by $f^{-1}(\tilde{\beta})$ in $\aleph$ by $\tilde{\mu}(\chi)=f^{-1}(\tilde{\beta})=\tilde{\beta}(f(\chi)), \forall \chi \in \aleph$.

Lemma34. Let $f$ be a homomorphism mapping from a KU-semigroup $(\aleph, *, \circ, 0)$ into a KUsemigroup $\left(\aleph^{\prime}, *^{\prime}, 0^{\prime}, 0^{\prime}\right)$. Then $\mathrm{f}^{\mathbf{- 1}}(\tilde{\beta})$ is an interval valued fuzzy $k$-ideal of $\boldsymbol{\aleph}$, if the mapping $\tilde{\beta}$ is an interval valued fuzzy $k$-ideal of $\aleph^{\prime}$.

Proof. For all $\chi \in \mathrm{N}$, we have $\tilde{\mu}(0)=\tilde{\beta}(\mathrm{f}(0)) \geq \tilde{\beta}(\mathrm{f}(\chi))=\tilde{\mu}(\chi)$. Let $, \gamma, \tau \in \mathrm{N}$, then we have $\tilde{\mu}(\chi * \tau)=\tilde{\beta}(f(\chi * \tau))=\tilde{\beta}\left(f(\chi) *^{\prime} f(\tau)\right) \geq \operatorname{rmin}\left\{\tilde{\beta}\left(f(\chi) *^{\prime}\left(f(\gamma) *^{\prime} f(\tau)\right)\right), \tilde{\beta} f(\gamma)\right\}$

$=r \min \{\tilde{\beta}(\mathrm{f}(\chi *(\gamma * \tau)), \tilde{\beta} \mathrm{f}(\gamma)\}$

$=r \min \{\tilde{\mu}(\chi *(\gamma * \tau)), \tilde{\mu}(\gamma)\}$

Also, we have $\tilde{\mu}(\chi \circ \gamma)=\tilde{\beta}(f(\chi \circ \gamma))=\tilde{\beta}(f(\chi) \circ f(\tau)) \geq \operatorname{rmin}\{\tilde{\beta}(f(\chi)), \tilde{\beta}(f(\gamma))\}$

$=r \min \{\tilde{\mu}(\chi)), \tilde{\mu}(\gamma)\}$.

Hence the proof is completed.

Theorem(35). Letf: $\aleph \rightarrow \aleph^{\prime}$ be an epimorphism between two KU-semigroups $\aleph$ and $\aleph^{\prime} . f(\tilde{\mu})$ is an interval valued fuzzy $k$-ideal of $\aleph^{\prime}$, if $\tilde{\mu}$ is an interval valued fuzzy $k$-ideal of $\aleph$.

Proof. Let $\chi^{\prime}, \gamma^{\prime} \in \mathcal{N}^{\prime}$, then $\exists \chi, \gamma \in \mathrm{N}$ such that $\mathrm{f}(\chi)=\chi^{\prime}$ and $\mathrm{f}(\gamma)=\gamma^{\prime}$. By definition of image, we have $f(\tilde{\mu})\left(\chi^{\prime}\right)=\sup \tilde{\mu}(\chi)_{\chi \in f^{-1}\left(\chi^{\prime}\right)}$, for some $\chi \in \aleph$, and,

$f(\tilde{\mu})\left(\gamma^{\prime}\right)=\sup \tilde{\mu}(\gamma)_{\gamma \in f^{-1}(\gamma)}$, for some $\gamma \in \aleph$.

We have $\tilde{\mu}(0) \geq \tilde{\mu}(\chi), \forall \chi \in \aleph$. Then,

(i) $\left.\left.f(\tilde{\mu})\left(0^{\prime}\right)=\sup \tilde{\mu}(0)\right)_{0 \in f^{-1}\left(0^{\prime}\right)} \geq \sup \tilde{\mu}(\chi)\right)_{\chi \in f^{-1}\left(\chi^{\prime}\right)}=f(\tilde{\mu})\left(\chi^{\prime}\right)$, for any $\chi^{\prime} \in \aleph$.

(ii) For any $\chi^{\prime}, \gamma^{\prime}, \tau^{\prime} \in \aleph^{\prime}$, let $\chi_{0} \in f^{-\mathbf{1}}\left(\chi^{\prime}\right), \gamma_{0} \in f^{-\mathbf{1}}\left(\gamma^{\prime}\right), \tau_{0} \in f^{-\mathbf{1}}\left(\tau^{\prime}\right)$, and since $f$ is ahomomorphism, then $f(\tilde{\mu})\left(\chi^{\prime} *^{\prime} \tau^{\prime}\right)=\sup \tilde{\mu}\left(\chi_{0} * \tau_{0}\right)_{\chi_{0} * \tau_{0} \in f^{-1}\left(\chi^{\prime} *^{\prime} \tau^{\prime}\right)}$

$$
\geq \operatorname{rmin}\left\{\sup \tilde{\mu}\left(\chi_{0} *\left(\gamma_{0} * \tau_{0}\right)\right)_{\left(\chi_{0} *\left(\gamma_{0} * \tau_{0}\right)\right) \in f^{-1}\left(\chi^{\prime} *^{\prime}\left(\gamma^{\prime} *^{\prime} \tau^{\prime}\right)\right)}, \sup \tilde{\mu}\left(\gamma_{0}\right)_{\gamma_{0} \in f^{-1}\left(\gamma^{\prime}\right)}\right\}
$$

$=r \min \left\{f(\tilde{\mu})\left(\chi^{\prime} *^{\prime}\left(\gamma^{\prime} *^{\prime} \tau^{\prime}\right)\right), f(\tilde{\mu})\left(\gamma^{\prime}\right)\right\}$

(iii) Forany $\chi^{\prime}, \gamma^{\prime} \in \boldsymbol{N}^{\prime}$, let $\chi_{0} \in f^{-\mathbf{1}}\left(\chi^{\prime}\right), \gamma_{0} \in f^{-1}\left(\gamma^{\prime}\right)$ be such that:

$f(\tilde{\mu})\left(\chi^{\prime} \circ \circ^{\prime} \gamma^{\prime}\right)=\sup \tilde{\mu}\left(\chi_{0} \circ \gamma_{0}\right)_{\chi_{0} \circ \tau_{0} \in f^{-1}\left(\chi^{\prime} \circ \tau^{\prime}\right)}$

$$
\geq \operatorname{rmin}\left\{\sup \tilde{\mu}\left(\chi_{0}\right)_{\left(\chi_{0}\right) \in f^{-1}\left(\chi^{\prime}\right)}, \sup \tilde{\mu}\left(\gamma_{0}\right)_{\gamma_{0} \in f^{-1}\left(\gamma^{\prime}\right)}\right\}
$$


$=r \min \left\{(\tilde{\mu})\left(\chi^{\prime}\right), f(\tilde{\mu})\left(\gamma^{\prime}\right)\right\}$

Hence the proof is completed.

Definition (36). If $\widetilde{\mu}$ and $\widetilde{\beta}$ are two interval valued fuzzysubsets of a set $\boldsymbol{\kappa}$. Then the product of $\tilde{\mu}$ and $\tilde{\beta}$ denoted by $\tilde{\mu} \times \tilde{\beta}$ is defined by:

$\tilde{\mu} \times \tilde{\beta}(\chi, \gamma)=r \min \{\tilde{\mu}(\chi), \tilde{\beta}(\gamma)\}$,for $\operatorname{all}(\chi, \gamma) \in \aleph \times \aleph$.

Theorem (37). The product $\tilde{\mu} \times \tilde{\beta}$ is an interval valued fuzzy $k$-ideal of $\aleph \times \aleph$, if $\tilde{\mu}$ and $\tilde{\beta}$ are interval valued fuzzy $k$-ideals of a KU-semigroup $\aleph$.

Proof. $\operatorname{Let}(\chi, \gamma) \in \aleph \times \aleph$, we have

$(\tilde{\mu} \times \tilde{\beta})(0,0)=\operatorname{rmin}\{\tilde{\mu}(0), \tilde{\beta}(0)\} \geq \operatorname{rmin}\{\tilde{\mu}(\chi), \tilde{\beta}(\gamma)\}=(\tilde{\mu} \times \tilde{\beta})(\chi, \gamma)$.

Now, let $\left(\chi_{1,}, \chi_{2}\right),\left(\gamma_{1}, \gamma_{2}\right),\left(\tau_{1}, \tau_{2}\right) \in \aleph \times \aleph$, then

$$
\begin{gathered}
\tilde{\mu} \times \tilde{\beta}\left[\left(\chi_{1} * \tau_{1}, \chi_{2} * \tau_{2}\right)\right]=\operatorname{rmin}\left\{\tilde{\mu}\left(\chi_{1} * \tau_{1}\right), \tilde{\beta}\left(\chi_{2} * \tau_{2}\right)\right\} \\
\geq \operatorname{rmin}\left\{\operatorname{rmin}\left\{\tilde{\mu}\left(\chi_{1} *\left(\gamma_{1} * \tau_{1}\right)\right), \tilde{\mu}\left(\gamma_{1}\right)\right\}, r \min \left\{\tilde{\beta}\left(\chi_{2} *\left(\gamma_{2} * \tau_{2}\right)\right), \tilde{\beta}\left(\gamma_{2}\right)\right\}\right\} \\
=\operatorname{rmin}\left\{\operatorname{rmin}\left\{\tilde{\mu}\left(\chi_{1} *\left(\gamma_{1} * \tau_{1}\right)\right), \tilde{\beta}\left(\chi_{2} *\left(\gamma_{2} * \tau_{2}\right)\right)\right\}, r \operatorname{rmin}\left\{\tilde{\mu}\left(\gamma_{1}\right), \tilde{\beta}\left(\gamma_{2}\right)\right\}\right\} \\
=\operatorname{rmin}\left\{(\tilde{\mu} \times \tilde{\beta})\left[\left(\chi_{1} *\left(\gamma_{1} * \tau_{1}\right)\right),\left(\chi_{2} *\left(\gamma_{2} * \tau_{2}\right)\right)\right],(\tilde{\mu} \times \tilde{\beta})\left(\gamma_{1}, \gamma_{2}\right)\right\} .
\end{gathered}
$$

And,

$$
\begin{aligned}
& \quad(\tilde{\mu} \times \tilde{\beta})\left(\chi_{1} \circ \chi_{2}\right)\left(\gamma_{1} \circ \gamma_{2}\right)=\operatorname{rmin}\left\{\tilde{\mu}\left(\chi_{1} \circ \chi_{2}\right), \tilde{\beta}\left(\gamma_{1} \circ \gamma_{2}\right)\right\} \\
& \geq \operatorname{rmin}\left\{\operatorname{rmin}\left\{\tilde{\mu}\left(\chi_{1}\right), \tilde{\mu}\left(\chi_{2}\right)\right\}, \operatorname{rmin}\left\{\tilde{\beta}\left(\gamma_{1}\right), \tilde{\beta}\left(\gamma_{2}\right)\right\}\right. \\
& =\operatorname{rmin}\left\{r \min \left\{\tilde{\mu}\left(\chi_{1}\right), \tilde{\beta}\left(\gamma_{1}\right)\right\}, r \min \left\{\tilde{\mu}\left(\chi_{2}\right), \tilde{\beta}\left(\gamma_{2}\right)\right\}\right\} \\
& =\operatorname{rmin}\left\{(\tilde{\mu} \times \tilde{\beta})\left(\chi_{1}, \gamma_{1}\right),(\tilde{\mu} \times \tilde{\beta})\left(\chi_{2}, \gamma_{2}\right)\right\} .
\end{aligned}
$$

Definition (38). Let $\tilde{\mu}$ be an interval valued fuzzy set in $\aleph$. If $\tilde{\mu}$ is defined by:

$\tilde{\mu}: S \times S \rightarrow D[0,1]$, then $\tilde{\mu}$ is named an interval valued fuzzy relation on a set $S$, where $S \subseteq \aleph$.

Definition (39). Let $\tilde{\beta}$ be an interval valued fuzzy set in $\aleph$. Then the strongest interval valued fuzzy relation on $\mathcal{N}$ by $\tilde{\beta}$ is denoted by $\tilde{\mu}_{\widetilde{\beta}}$ and defined as follows $\tilde{\mu}_{\widetilde{\beta}}(\chi, \gamma)=$ $\operatorname{rmin}\{\tilde{\beta}(\chi), \tilde{\beta}(\gamma)\}$, for all $\chi, \gamma \in \aleph$.

Lemma (40). If the strongest interval valued fuzzy relation on $\aleph$ is an interval valued fuzzy $k$-ideal of $\aleph \times \aleph$, then $\tilde{\beta}(\chi) \leq \tilde{\beta}(0)$, for all $\chi \in \aleph$ and $\tilde{\beta}$ is an interval valued fuzzy set of a KU-semigroup $\aleph$.

Proof. Let $\tilde{\mu}_{\widetilde{\beta}}$ be an interval valued fuzzy $k$-ideal of $\aleph \times \aleph$, it follows that:

$\tilde{\beta}(\chi)=\operatorname{rmin}\{\tilde{\beta}(\chi), \tilde{\beta}(\chi)\}=\tilde{\mu}_{\widetilde{\beta}}(\chi, \chi) \leq \tilde{\mu}_{\widetilde{\beta}}(0,0)=\operatorname{rmin}\{\tilde{\beta}(0), \tilde{\beta}(0)\}=\tilde{\beta}(0)$.

Hence, $\tilde{\beta}(\chi) \leq \tilde{\beta}(0)$. 
Ibn Al-Haitham Jour. for Pure \& Appl. Sci. 33 (2) 2020

Theorem(41). The strongest interval valued fuzzy relation $\tilde{\mu}_{\widetilde{\beta}}$ on $\aleph$ is an interval valued fuzzy $k$-ideal of $\aleph \times \aleph$ iff the mapping $\tilde{\beta}$ is an interval valued fuzzy $k$-ideal of $\aleph$.

Proof. $(\Leftarrow)$ Since $\tilde{\beta}$ is an interval valued fuzzy $k$-ideal of $\aleph$, then $\tilde{\mu}_{\widetilde{\beta}}(0,0)=$ $\operatorname{rmin}\{\tilde{\beta}(0), \tilde{\beta}(0)\}=\operatorname{rmin}\{\tilde{\beta}(\chi), \tilde{\beta}(\gamma)\}=\tilde{\mu}_{\widetilde{\beta}}(\chi, \gamma)$.

Now, for any $\left(\chi_{1}, \chi_{2}\right)\left(\gamma_{1}, \gamma_{2}\right)\left(\tau_{1}, \tau_{2}\right) \in \aleph \times \aleph$, we have:

$$
\begin{gathered}
\tilde{\mu}_{\widetilde{\beta}}\left(\chi_{1}, * \tau_{1}, \chi_{2} * \tau_{2}\right)=\operatorname{rmin}\left\{\tilde{\beta}\left(\chi_{1} * \tau_{1}\right), \tilde{\beta}\left(\chi_{2} * \tau_{2}\right)\right\} \\
\geq \operatorname{rmin}\left\{\operatorname{rmin}\left\{\tilde{\beta}\left(\chi_{1}, *\left(\gamma_{1} * \tau_{1}\right)\right), \tilde{\beta}\left(\gamma_{1}\right)\right\}, \operatorname{rmin}\left\{\tilde{\beta}\left(\chi_{2} *\left(\gamma_{2} * \tau_{2}\right)\right), \tilde{\beta}\left(\gamma_{2}\right)\right\}\right. \\
=\operatorname{rmin}\left\{\operatorname{rmin}\left\{\tilde{\beta}\left(\chi_{1} *\left(\gamma_{1} * \tau_{1}\right)\right), \tilde{\beta}\left(\chi_{2} *\left(\gamma_{2} * \tau_{2}\right)\right)\right\}, r \operatorname{rmin}\left\{\tilde{\beta}\left(\gamma_{1}\right), \tilde{\beta}\left(\gamma_{2}\right)\right\}\right. \\
=\operatorname{rmin}\left\{\tilde{\mu}_{\widetilde{\beta}}\left(\chi_{1} *\left(\gamma_{1} * \tau_{1}\right)\right),\left(\chi_{2}, *\left(\gamma_{2} * \tau_{2}\right)\right), \tilde{\mu}_{\widetilde{\beta}}\left(\gamma_{1}, \gamma_{2}\right)\right\} .
\end{gathered}
$$

And,

$$
\begin{aligned}
\tilde{\mu}_{\widetilde{\beta}}\left[\left(\chi_{1}, \circ \chi_{2}\right),\left(\gamma_{1} \circ \gamma_{2}\right)\right] & =\operatorname{rmin}\left\{\tilde{\beta}\left(\chi_{1} \circ \chi_{2}\right), \tilde{\beta}\left(\gamma_{1} \circ \gamma_{2}\right)\right\} \\
\geq & \operatorname{rmin}\left\{\operatorname{rmin}\left\{\tilde{\beta}\left(\chi_{1},\right), \tilde{\beta}\left(\chi_{2}\right)\right\}, r \min \left\{\tilde{\beta}\left(\gamma_{1}\right), \tilde{\beta}\left(\gamma_{2}\right)\right\}\right\} \\
= & \operatorname{rmin}\left\{\tilde{\mu}_{\widetilde{\beta}}\left(\chi_{1}, \chi_{2}\right), \tilde{\mu}_{\widetilde{\beta}}\left(\gamma_{1}, \gamma_{2}\right)\right\} .
\end{aligned}
$$

$(\Rightarrow)$ For all, $(\chi, \gamma) \in \aleph \times \aleph$,

$\operatorname{rmin}(\tilde{\beta}(0), \tilde{\beta}(0))=\tilde{\mu}_{\widetilde{\beta}}(0,0) \geq \tilde{\mu}_{\widetilde{\beta}}(\chi, \gamma)=\operatorname{rmin}\{\tilde{\beta}(\chi), \tilde{\beta}(\gamma)\}$.

Then $\tilde{\beta}(0) \geq \tilde{\beta}(\chi), \forall \chi \in \aleph$.

Now, let $\left(\chi_{1}, \chi_{2}\right)\left(\gamma_{1}, \gamma_{2}\right)\left(\tau_{1}, \tau_{2}\right) \in \aleph \times \aleph$.

Then

$$
\begin{aligned}
& \operatorname{rmin}\left(\tilde{\beta}\left(\chi_{1} * \tau_{1}\right), \tilde{\beta}\left(\chi_{2} * \tau_{2}\right)\right\}=\tilde{\mu}_{\widetilde{\beta}}\left(\chi_{1} * \tau_{1}, \chi_{2} * \tau_{2}\right) \\
& \geq \operatorname{rmin}\left\{\left(\tilde{\mu}_{\widetilde{\beta}}\left(\chi_{1} * \tau_{1}\right), \tilde{\mu}_{\widetilde{\beta}}\left(\chi_{2} * \tau_{2}\right)\right\}\right. \\
& =\operatorname{rmin}\left\{\operatorname{rmin}\left\{\tilde{\mu}_{\widetilde{\beta}}\left(\chi_{1} *\left(\gamma_{1} * \tau_{1}\right)\right), \tilde{\mu}_{\widetilde{\beta}}\left(\gamma_{1}\right)\right\}, r \min \left\{\tilde{\mu}_{\widetilde{\beta}}\left(\chi_{2} *\left(\gamma_{2} * \tau_{2}\right)\right), \tilde{\mu}_{\widetilde{\beta}}\left(\gamma_{2}\right)\right\}\right\} \\
& \operatorname{rmin}\left\{r \min \left\{\tilde{\mu}_{\widetilde{\beta}}\left(\chi_{1} *\left(\gamma_{1} * \tau_{1}\right)\right), \tilde{\mu}_{\widetilde{\beta}}\left(\chi_{2} *\left(\gamma_{2} * \tau_{2}\right)\right)\right\}, r m i n\left\{\tilde{\mu}_{\widetilde{\beta}}\left(\gamma_{1}\right), \tilde{\mu}_{\widetilde{\beta}}\left(\gamma_{2}\right)\right\}\right. \\
& \left.=\operatorname{rmin}\left\{\operatorname{rmin} \tilde{\mu}_{\widetilde{\beta}}\left\{\left(\chi_{1} *\left(\gamma_{1} * \tau_{1}\right)\right),\left(\chi_{2} *\left(\gamma_{2} * \tau_{2}\right)\right)\right\}, \tilde{\mu}_{\widetilde{\beta}}\left(\gamma_{1}, \gamma_{2}\right)\right\}\right\} \\
& =\min \left\{\operatorname{rmin}\left\{\tilde{\beta}\left(\chi_{1} *\left(\gamma_{1} * \tau_{1}\right)\right), \tilde{\beta}\left(\chi_{2} *\left(\gamma_{2} * \tau_{2}\right)\right)\right\}, r \operatorname{rmin}\left\{\tilde{\beta}\left(\gamma_{1}\right), \tilde{\beta}\left(\gamma_{2}\right)\right\}\right. \\
& =\operatorname{rmin}\left\{\operatorname { r m i n } \left\{\tilde{\beta}\left(\chi_{1} *\left(\gamma_{1} * \tau_{1}\right), \tilde{\beta}\left(\gamma_{1}\right)\right\}, r \operatorname{rin}\left\{\tilde{\beta}\left(\chi_{2} *\left(\gamma_{2} * \tau_{2}\right), \tilde{\beta}\left(\gamma_{2}\right)\right\}\right.\right.\right.
\end{aligned}
$$

In particular, if we take $\chi_{2}=\gamma_{2}=\tau_{2}=0$, then $\tilde{\beta}\left(\chi_{1} * \tau_{1}\right) \geq r \min \left\{\tilde{\beta}\left(\chi_{1} *\left(\gamma_{1} * \tau_{1}\right), \tilde{\beta}\left(\gamma_{1}\right)\right\}\right.$. Hence, the proof is completed.

\section{Conclusion}

The concept of an interval value fuzzy set of KU-semigroup is introduced and some related properties are investigated. Also, some types of interval value fuzzy ideals are studied and the relationship between them is stated. Then, an interval value fuzzy $k$-ideal of KUsemigroup is studied and a few properties are obtained. Furthermore, the notion of a 
homomorphism is discussed. Main purpose of our future work is to investigate fuzzy of several types of ideals with special properties such as an intuitionistic fuzzy and hyper of KUsemigroup.

\section{References}

1. Prabpayak, C.; Leerawat, U. On ideals and congruence in KU-algebras.Scientia Magna Journal.2009, 5, 1, 54-57.

2. Prabpayak, C.; Leerawat, U. On isomorphisms of KU-algebras.Scientia Magna journal.2009, 5, 3, 25-31.

3. Kareem,F.F.;Hasan, E. R.On KU-semigroups.International Journal of Science and Nature.2018, 9, 1, 79-84.

4. Zadeh, L.A. Fuzzy Sets.Inform and Control.1965, 8, 338-353.

5. Mostafa, S.M.; Abd-Elnaby, M.A.; Yousef, M.M.M. Fuzzy ideals of KU-Algebras. International Mathematical Forum.2011, 6, 63, 3139-3149.

6. Hasan, E.R.; Kareem, F.F. Fuzzy KU-Semi-Groups and Investigate Some Basic Properties.Journal of Engineering and Applied Science.2018, 13, 18, 7739-7744.

7. Akram, M.; Yaqoob, N.; Kavikumar, J. Interval valued $(\widetilde{\theta}, \widetilde{\delta})$-fuzzy KU-ideals of KUalgebras.Int. J. Pure Appl. Math.2014, 92, 3, 335-349.

8. Lee, K.M. Bipolar-valued fuzzy sets and their operations. Proc. Int. Conf. onIntelligent Technologies, Bangkok, Thailand.2000, 307-312.

9. Lee, K.M. Comparison of interval-valued fuzzy sets, intuitionistic fuzzy sets, and bipolar-valued fuzzy sets.J. Fuzzy Logic Intelligent Systems.2004, 14, 125-129.

10. Mostafa, S.M.; Abd-Elnaby, M.A.; Elgendy, O. R. Interval-valued Fuzzy KU ideals in KU-algebras.Int. Math. Forum.2011, 6, 64, 3151-3159.

11. Mostafa, S.M.; Radwan, A.E.; Ibrahem, F.A.; Kareem, F.F. Interval value fuzzy n-Fold KU-ideals of KU-algebras.J. Math.Comput.Sci.2015, 5, 2, 246-264.

12. Saeid, A.B. Bipolar-valued fuzzy BCK\BCI-algebras. World Applied Sciences Journal.2009, 7, 11, 1404-1411. 